\title{
Publisher's Note: Quantum vortices and thermally induced luminescence of nitrogen nanoclusters immersed in liquid helium [Phys. Rev. B 95, 104502 (2017)]
}

\author{
A. Meraki, P. T. McColgan, P. M. Rentzepis, R. Z. Li, D. M. Lee, and V. V. Khmelenko $\odot$ \\ (Q) (Received 6 November 2020; published 20 November 2020)
}

DOI: 10.1103/PhysRevB.102.179902

This paper was published online on 1 March 2017 with an omission of an Acknowledgment statement. The Acknowledgment section should read "This work was supported by NSF Grant No. DMR 1209255, Welch Foundation Grant No. A 1884, and AFOSR Grant No. FA9550-18-1-0100." The paper has been corrected as of 9 November 2020. The Acknowledgment is not present in the printed version of the journal. 